\title{
SOBRE EL ESTADO Y LAS UNIVERSIDADES
}

\author{
Diálogo* CON Fernando Atria**
}

\section{¿Cuál cree usted que ha sido históricamente la relación entre el Estado y la Universidad?}

La pregunta asume que ha habido, históricamente, una respuesta a esta pregunta. Por mi parte, yo creo que una de las cuestiones más importantes para nosotros es entender que hoy estamos viviendo la culminación de un proceso de redefinición drástica de esta relación. Hay varias causas detrás de este proceso, de las cuales hay dos que deben ser destacadas. Una es el cambio en la función que las instituciones que llamamos "universidades" cumplen, de la que hablaremos más adelante. La otra, que es la que quiero enfatizar ahora, es la redefinición de la idea de "lo público" en el contexto de la cual ocurre este proceso.

Hasta las reformas de 1980, la educación universitaria era vista como algo que no estaba sujeto al mercado, porque era intrínseca a ella una dimensión pública. La educación universitaria era educación "pública" incluso cuando las instituciones eran jurídicamente privadas, porque lo "público" de la actividad universitaria estaba dado no por la naturaleza del agente que la proveía sino por la comprensión de la actividad misma. Por eso, incluso hoy, más de tres décadas después de esas reformas, cuando hablamos de universidades "privadas" no solemos hacer referencia a las universidades creadas antes de 1980, incluso a las privadas. Desde el punto de vista de las distinciones que hoy día son importantes, es bastante claro que, por ejemplo, la Universidad Austral y la Universidad de Concepción están más cerca de la Universidad de Chile que de la Universidad del Desarrollo o la Universidad de las Américas, pese a que estas cuatro universidades son igualmente "privadas", en el sentido de no-estatales. Esto porque la forma en que las universidades no estatales anteriores a 1980 se entienden a sí mismas y entienden su función está más cercana a las universidades estatales que a las universidades privadas creadas después de 1980. De hecho incluso entre las universidades creadas después de 1980 es posible hacer una distinción lo que podría llamarse la "primera ola" de universidades privadas, todavía en algún sentido bajo el influjo de la comprensión pre-1980 (la Universidad Diego Portales y la Universidad Central), y las creadas desde los 90, cuando esa comprensión ya no era dominante (la Universidad Gabriela Mistral escapa a esta observación).

Esa comprensión, como ya está dicho, entendía a la universidad como una institución intrínsecamente pública. Esta compresión pública de la idea de universidad se ha invertido hoy, de modo que hoy la institución universitaria es pensada como una institución intrínsecamente privada. Entonces, más que la relación entre el Estado y la universidad, lo que cambió fue la

Realizada el 26 de junio de 2012. Interlocutores: José Luis Castro y Nicolás López Pérez.

** PhD, Universidad de Edimburgo. Abogado, Licenciado en Ciencias Jurídicas y Sociales, Universidad de Chile. Profesor regular titular, Universidad Adolfo Ibáñez; Profesor asociado, Facultad de Derecho, Universidad de Chile 
comprensión de la actividad universitaria en su relación con lo público. Y claro, eso necesariamente tenía que tener impacto en la relación en sentido estricto del Estado con sus universidades, porque ese es el caso más obvio: las universidades estatales. Lo que ha pasado desde 1980 en adelante es que el Estado ha tendido a relacionarse con sus universidades negando que sean universidades distintas a las universidades privadas; entonces, por ejemplo, las universidades estatales se financian básicamente de la misma manera que las universidades privadas, esto es, con aranceles pagados por los estudiantes (es cierto, tienen aportes directos, pero esos aportes en relevancia han disminuido sistemáticamente).

Pero como está dicho no es sólo una transformación de la idea de universidad, porque lo que se transformó fue la idea de lo público. Así, por ejemplo, lo mismo puede decirse de la televisión pública. Hoy la televisión pública no tiene un régimen distinto al de la televisión privada en cuanto a su modo de operación o su forma de financiamiento. Y la regla aquí es "dime como te financias y te diré como actuarás": es ingenuo pensar que si un canal de televisión se financia como una actividad privada, el solo hecho de que sea del Estado va a hacer que desempeñe una función distinta. Y por eso hoy en cuanto a su función, al tipo de periodismo que realiza y a las finalidades que su programación persigue no hay diferencia entre el canal del Estado y los canales privados de televisión. Es el mismo proceso que ha afectado a las universidades estatales, aunque el hecho de que entre estas exista una que tiene 150 años de tradición ha permitido que el proceso de privatización de la autocomprensión de la institución haya sido menos completo. Pero también son instituciones que se financian como instituciones privadas, y por eso ha sido posible, en la discusión sobre las universidades que en los hechos funcionan con fines de lucro, alegar que en las universidades estatales también hay fines de lucro. Esto es, por supuesto, absurdo, porque el estado no retira utilidades de sus universidades. Lo que se acusa aquí es una estrategia que las universidades estatales han debido adoptar para enfrentar el hecho de que sus condiciones de operación y financiamiento son en todo las de una universidad privadas, salvo en el hecho de que su régimen interno es el del derecho administrativo, lo que les impide competir.

Para volver a la pregunta, es importante notar que el problema no es que el estado haya o no asumido su relación especial respecto de sus universidades. El problema heredado de 30 años de neoliberalismo es mucho más grave que eso, porque el problema es que hoy parece ser difícil de articular una respuesta a la pregunta de qué hay en lo público salvo el dato formal de la propiedad del Estado.

\section{¿Vale hablar respecto de una conceptualización de educación superior en el contexto de hoy en día?}

El sentido por el cual todavía es importante hacer la distinción entre educación superior -en el sentido de postsecundaria- y el resto es que la educación post secundaria no es obligatoria, y yo creo que eso afecta el régimen de esa educación. Por supuesto, con esto no estoy diciendo que haya necesariamente algo en la educación post-secundaria que sea esencialmente distinto de la educación secundaria. En el pasado la educación secundaria no era obligatoria, 
y lo era solo la primaria. Hoy entendemos que el derecho a la educación exige educación secundaria, pero no post-secundaria. Quizás en el futuro nuestra comprensión del derecho a la educación se extienda hasta cubrir la educación terciaria. Sólo estoy diciendo que si eso ocurre entonces algo importante habrá cambiado, porque hoy no lo entendemos así.

Esto, por supuesto, no quiere decir que en la discusión sobre educación superior no esté involucrado el derecho a la educación, que éste tenga que ver solamente con la educación escolar y no con la superior. Sería absurdo entenderlo asi., Pero notar que eso sería un absurdo no puede llevar a negar la diversa forma que asume el derecho a la educación cuando se trata de educación escolar y educación superior, lo que justifica seguir manteniendo esta última categoría.

Supongo que no digo nada especialmente polémico al decir que hoy es una situación inaceptable que haya personas que no acceden a la educación secundaria y más aun primaria. Esto quiere decir: nuestra manera de comprender el contenido del derecho a la educación, en lo que se refiere a la educación escolar es que ella es necesaria: infringe el derecho a la educación el que no haya educación para todos y cada uno. Pero esto no es así tratándose de educación superior: no necesariamente es una situación flagrantemente inaceptable que la cobertura de la educación superior no alcance el $100 \%$ de la cohorte respectiva. En lo que se refiere a la educación superior, el contenido del derecho a la educación no es que ella es necesaria para todos y cada uno, sino que ella debe estar disponible para todos. Esto implica que el régimen de la educación superior tiene que estar orientado, más que a su expansión hasta alcanzar cobertura completa, a abrirle oportunidades a todo aquel que quiera acceder a ella. Esta diferencia notoria con la educación escolar hace que debamos pensar en la educación superior de modo distinto a la educación escolar, y en ese sentido la categoría de educación superior o postsecundaria es una categoría que todavía es importante mantener.

Esto no significa, por supuesto, que no haya relaciones entre ambas: no es que lo que digamos acerca de la educación superior no tenga consecuencia alguna respecto de la educación escolar y viceversa, porque las tiene y son muchas, pero a pesar de eso todavía hay aspectos en los cuales el régimen de la educación postsecundaria puede ser distinto de la educación escolar. A mi juicio, un ejemplo es el provisto por la idea de selección. Por razones que no puedo explicar ahora, yo creo que toda forma de selección debe ser completamente abolida de la educación escolar. ¿Debe ser igualmente abolida de la educación superior? A mi juicio la respuesta aquí debe ser más matizada, y tomar en cuenta aspectos que tratándose de educación escolar son inaceptables (como el interés público en tener universidades estatales de excelencia, por ejemplo, que fijen un estándar para el desarrollo de la idea de institución universitaria).

¿Qué influencia puede tener la dicotomía que se establece entre Universidades públicas y privadas de cara al desarrollo futuro del sistema universitario?

Para volver a una idea mencionada al principio, una de las cosas más importantes que es consecuencia de estos 30 años de neoliberalismo es que la idea de lo público ha ido perdiendo 
contenido. En otras palabras, nuestro problema no es que se hayan privatizado las sanitarias, los puertos, etc. sino que se ha ido privatizando el Estado, en el sentido de que hoy pensamos sobre el Estado como si fuera una agente que se diferencia del privado sólo porque es más grande. Ello es particularmente evidente en materia escolar: pensamos el sistema educacional desde el punto de vista del colegio particular pagado, y entonces entendemos el colegio particular subvencionado como uno particular pagado con la única diferencia de que la colegiatura la paga el Estado mediante la subvención, y luego entendemos el colegio municipal como uno particular subvencionado con la única diferencia de que su sostenedor es una municipalidad.

Esto implica que en nuestras circunstancias el concepto de lo público ya no puede darse por sentado. No es suficiente reclamar, por ejemplo, por el fortalecimiento de la educación pública, porque eso hoy significa fortalecimiento de la educación provista por escuelas de dependencia municipal. Y la pregunta obvia es: ¿qué tiene de especial la educación cuando la provee una escuela de dependencia municipal? ¿Por qué la educación provista por escuelas de dependencia municipal debe ser fortalecida? ¿Por qué sería grave que las escuelas de dependencia municipal declinaran en número hasta terminar siendo marginales, en la medida en que escuelas de otras dependencias surgieran para ofrecer las plazas respectivas?

Por supuesto, con lo anterior no estoy sugiriendo que no haya respuestas a esas preguntas. Yo creo que las hay, y buenas (en el sentido de: correctas). Todo lo que estoy sugiriendo es que hay que tener respuesta para esas preguntas cuando uno quiere luchar por el fortalecimiento de la educación pública. Esto es lo que quiero decir al decir que hoy, después de 30 años de neoliberalismo, no podemos dar la idea de "educación pública" por sentada.

Como la idea de "lo público" no puede hoy ser dado por sentado, debemos volver cándidamente al principio y articular alguna idea de lo público que muestre lo que hoy no es evidente: por qué es importante. Por qué es importante la educación pública, y qué sería grave de que ella continuara el proceso de decadencia en que está sumida. Hoy "educación pública” en particular y "lo público" en general es una categoría formal, que se refiere a lo que algunos, para enfatizar su irrelevancia, llaman "la naturaleza jurídica" del agente. Pero las categorías formales nunca pueden dar cuenta de sí mismas, nunca pueden responder la pregunta de por qué la distinción que ellas trazan es una distinción importante. Dar cuenta de la categoría de "educación pública" es algo que debemos hacer políticamente, a través de la discusión y acción política. Es necesario volver a aprender lo importante que es lo público.

Dicho de otro modo, parte de lo que significa hoy en día preguntarse por lo público tanto en educación como en otras áreas- es volver a la pregunta sustantiva que está detrás de las categorías formales. Al hacerlo, es evidente que la referencia a la propiedad estatal no nos dará pista alguna, porque la pregunta será sobre substancia y no sobre forma: ¿por qué es importante la educación pública, o la televisión pública? Nuestra respuesta a esa pregunta es una caracterización substantiva de lo público: es importante la educación pública porque cumple una función determinada, por lo que la educación que cumple esa función es la educación pública. 
Armados de este concepto substantivo, deberemos volver sobre las categorías formales. Al hacer esto podemos notar dos cosas: la primera es hay educación formalmente pública (provista por establecimientos dependientes de municipalidades) que no es substantivamente pública (no cumple la función pública), y podremos encontrar que establecimientos formalmente privados pueden desempeñar esa función pública. Si este es el caso, como yo creo que es (eso lo he argumento latamente en dos artículos), entonces la manera de defender la educación pública hoy no es reducible a la defensa de la educación municipal (o a su desmunicipalización, de hecho), sino debe adoptar una forma que en algún sentido es más radical y en otro menos: toda la educación debe ser pública en el sentido de que debe cumplir la función pública, aun cuando sea provista por entidades jurídicamente "privadas". En esto, la situación actual de las universidades, donde esta distinción entre universidades privadas como la Universidad de Concepción pero que se entienden a sí mismas como públicas y universidades enteramente privadas, nos da una pista invaluable. Debemos pensar cómo configurar un "régimen de lo público" que asegure que todo el que se sujeta a él cumple la función pública relevante, y luego decir que todo el que esté dispuesto a participar de la actividad bajo ese régimen es bienvenido.

Sólo para ejemplificar haciendo referencia breve a un argumento que no puedo desarrollar ahora, yo creo que lo que es importante acerca de la educación (escolar, por las razones ya dadas) pública es que ella es provista por instituciones que ofrecen incondicionalmente un servicio educacional al ciudadano. La idea substantiva de "educación pública" excluye, a mi juicio, toda posibilidad, por parte del establecimiento, de formular unilateralmente condiciones que sólo algunos ciudadanos pueden satisfacer, porque todos los ciudadanos tienen el mismo derecho a la educación, y la posibilidad de elegir es de todos por igual. La educación substantivamente privada es la que es ofrecida conforme al régimen del mercado, es decir, conforme a un régimen en que cada parte contratante puede poner las condiciones que desee para contratar. Hoy muchos establecimientos formalmente públicos (los liceos que la prensa denomina "emblemáticos" y los "de excelencia", entre otros) son en este sentido establecimientos privados, aun cuando dependen de municipalidades, y algunos establecimientos privados que no ponen condiciones son en este sentido educación pública. La coexistencia de educación substantivamente pública y educación substantivamente privada genera segregación, y tiende a transformar a la primera en un guetto de marginalidad y exclusión. La conclusión es que en este sentido substantivo toda educación ha de ser pública, lo que sin embargo no prejuzga si los proveedores (los establecimientos) deben ser formalmente públicos, es decir órganos del Estado o dependientes de éstos. A mi juicio el "fortalecimiento de la educación pública" no consiste en invertir más recursos en la educación formalmente pública, sino en crear un régimen legal en virtud del cual todos los establecimientos educacionales sean substantivamente públicos aun cuando haya un "sector privado" en el sentido formal del término.

Algo similar puede decirse de la educación universitaria, con la ventaja de que aquí esta idea de que hay instituciones que cumplen una función pública aun cuando sean privadas existe en la distinción entre universidades "del CRUCH" y las demás. Aquí la dimensión substantiva- 
mente pública de las universidades se ha manifestado, entre otras cosas, en que ellas entienden que el problema de la admisión no es un problema privado de cada institución, sino algo que debe ser tematizado a un nivel superior a las instituciones. Esta es la idea en que descansa el sistema unificado de admisión de las universidades del CRUCH. Dicho sistema de admisión es una manera de enfatizar que las universidades que participan de él no se entienden como agentes de mercado que pueden poner unilateralmente condiciones a la contraparte, 'por lo que la decisión sobre cuáles son los criterios de admisión no puede ser tomada unilateralmente por cada institución, lo que a su vez implica que quien defiende un nuevo sistema o criterio de admisión deberá justificar frente a las demás instituciones no que dicho sistema o criterio es conveniente para esa universidad, sino para todas.

Con respecto al tema del financiamiento en las universidades, últimamente se ha llevado a la palestra el debate de si el Estado debería financiar a estas universidades privadas, porque, se dice, el financiamiento debe seguir al estudiante y no a la institución. ¿Cree usted que debería darse esa lógica de financiamiento a las universidades privadas?

Si uno toma el sistema universitario actual como existe, con un sistema de acreditación bastante débil y una fiscalización también extraordinariamente débil por no decir inexistente, entonces sugerir un sistema integrado de financiamiento al estudiante, con independencia de la institución en la que estudie, pareciera un sinsentido, porque parece sólo una manera de mejorar el negocio para algunas universidades que podrán desentenderse del problema de tener que cobrar a sus estudiantes porque tendrán una confiable caja pagadora. Aunque esto es verdad, yo creo, sin embargo, que hay más aspectos que deben ser tomados en consideración.

En alguna medida, cuando el Estado financia, está publificando lo que financia, porque si el Estado financia una actividad es porque hay un interés público en que esa actividad sea financiada. Entonces, aunque un sistema integrado de financiamiento pareciera que es una medida que va en beneficio del negocio de las universidades privadas, tiene en sí la implicación evidente de que si el Estado financia a esas universidades ellas deberán estar sujetas a lo que más arriba denominaba un "régimen de lo público". Es precisamente por eso que es tan importante, hoy, preguntarse sobre ese régimen (qué implica, por ejemplo, respecto de la selección de estudiantes, del régimen de selección de profesores, de sus formas de gobierno interno, etc). A mi juicio, la existencia de un sistema integrado de financiamiento va a hacer ineludibles estas preguntas. Por esto a mi me ha sorprendido que la idea de un sistema integrado haya sido plateada por este gobierno. Yo creo que lo que explica esto es que no se han dado cuenta de que al dar ese paso lo que antes era simplemente un acuerdo de privados en el mercado (yo voy al mercado y compro lo que quiero) está siendo publificado, y al ser publificado se plantea el espacio para decir que la actividad universitaria es una actividad que debería ser siempre pública, no siempre estatal, como decíamos antes, pero sí siempre pública. Por eso, yo no me opondría a este proyecto de financiamiento aunque abogaría por su radicalización. Es decir, tomaría la idea que este gobier- 
no ha introducido en la discusión: - que hay un interés público en la actividad universitaria - y dejaría que ella se desarrollara conforme a su propia lógica, que llega mucho más allá y en otras direcciones de lo que el gobierno o espera.

El proyecto del gobierno supone que la educación universitaria no es algo que se compra en el mercado sino que hay un interés público en que todos puedan acceder a ella, lo que niega los términos fundamentales de la idea neoliberal que ha informado la acción del Estado durante los últimos 30 años. A mi juicio, esta propuesta consecuencia de un cambio extraordinariamente importante que ocurrió en las movilizaciones estudiantiles de 2011, en que lo que hasta entonces era de sentido común fue objetado por primera vez de modo frontal y directo. Claro, lo que hoy estamos discutiendo es el nuevo sentido común interpretado por un gobierno que está en contra de él, y por eso en el proyecto del gobierno hay mucho que corregir y la idea en la que descansa se manifiesta limitada arbitrariamente de muchos modos. Pero está ahí, y ahora hay que empujarla y fomentar que ella se desarrolle conforme a su propia lógica interna.

Se ha dado al interior de la opinión pública la idea respecto de que el Estado tiene que tender al bien común, la educación como un derecho fundamental debe ser bien regulada por el Estado para un buen funcionamiento de las instituciones general. ¿Cuál es, en este sentido, la función del Estado?

Bueno, yo creo que la regulación de nuestro sistema universitario es una regulación que se dictó cuando el sentido común indicaba que el deber del Estado es garantizar un estándar mínimo y que sobre ese mínimo cada uno ve cómo se las arregla. Entonces, en materia de calidad universitaria el problema del Estado no era adoptar políticas que tendieran a que las universidades tendieran a tener todas una calidad importante, sino que era establecer un mínimo. Y yo creo que el sistema de acreditación como existe hoy día va en esa línea, o sea, simplemente pretende excluir, no acreditar a las que están en la peor situación, levantarlas hasta un mínimo y una vez que estén sobre ese mínimo no hay problema. Yo creo que lo que pasó el año pasado es una impugnación directa a esa idea del deber del Estado, es lo que en algunos de los discursos de los estudiantes aparecía como una objeción al Estado subsidiario. Lo importante es la idea que está detrás de ello, que es impugnar esta idea de que el Estado tiene por función garantizar mínimos (en educación, en pensiones, en salud, en la manera en que el Estado se entiende en general) y pasar a una en la que el Estado tenga por función crear sistemas que tiendan hacia provisión igualitaria en algunos aspectos de la vida de las personas. Si uno piensa de ese modo, o sea, que el deber del Estado no es garantizar mínimos sino que es asegurar en ciertas dimensiones de la vida provisión igualitaria, entonces la pregunta por la acreditación cambia, porque ya no se trata de garantizar mínimos sino que de lograr que cada universidad sea la mejor universidad que puede ser. Hoy día hay bastante consenso en que el sistema de acreditación tal como existe hoy en día solo permite cazar al más deficitario (y a veces ni siquiera eso), yo creo que eso también va a cambiar. 
Por eso es bien importante la manera en que el sentido común ha cambiado, y cuando cambia el sentido común todas estas cosas empezar a ser discutidas en otros términos, y eso es lo más importante que está pasando en educación, es algo cuyos efectos no se va a notar ahora con la ley de superintendencia que se va a aprobar en Agosto, sino que se va a entender que todas las discusiones en educación de ahora en adelante van a ser entendidas en otros términos. Está cambiando el sentido común, o en otros términos, la hegemonía de ciertas ideas y esa es una cuestión que le permite ser a uno razonablemente optimista.

En torno a la lógica de un Estado regulador y vislumbrando en un largo plazo ¿existiría una relación tripartita entre Estado, universidad y mercado? y dentro de esa misma lógica ¿la mercantilización de la educación viene a ser una tendencia que va a crecer sostenidamente en el tiempo, transformando a la universidad en un agente de mercado?

Es bien difícil pensar que va a ser posible volver a una situación en la cual la educación superior -o la educación escolar para el caso- sea predominantemente estatal. En esas condiciones, la pregunta que sugería más arriba (¿qué hay de importante en que la educación la provean órganos estatales?) se hace urgente. La respuesta sugerida era que es importante la provisión pública porque es importante que la educación no se provea por agentes de mercado. El problema, claro, es que hoy órganos del Estado se comportan como agentes de mercado. Los ejemplos mencionados más arriba eran Televisión Nacional de Chile y las universidades públicas en general, en la medida en que prácticamente todo su ingreso proviene de lo que pagan los estudiantes. Ahora bien, el hecho de que haya órganos del Estado que operan como agentes de mercado plantea de manera más enfática el problema central: el Estado se ha privatizado, de modo que ya no vemos problema en que el Estado actúe como un agente de mercado. Y entonces, si lo que importaba de la educación pública era que fuera provista por entidades que no fueran agentes de mercado, nos encontramos con que el hecho de que las universidades sean o no estatales no es una garantía. Y entonces hay que dar vuelta la pregunta y partir preguntándose: ¿por qué es importante que las universidades no sean agentes de mercado?

A mi juicio, es importante responder esta pregunta correctamente, porque el mercado tiene hoy en día dos dimensiones: el mercado en un sentido es el espacio donde yo puedo elegir, donde lo que recibo es lo que quiero recibir, sin estar obligado por vínculos tradicionales, por el lugar en que nací, etc. No tengo vínculos anteriores a mi voluntad respecto a qué es lo que debo hacer con mi vida. En este sentido, entonces, el mercado es efectivamente un espacio de libertad que nos libera de esos vínculos tradicionales y preexistentes. Como dijo Maine, la irrupción del mercado en este sentido fue emancipatoria, porque significó el paso del estatus al contrato. No es en este sentido que es problemático que las universidades sean agentes de mercado. No se trata de que la posibilidad de los estudiantes de elegir lo que quieren estudiar o la institución en que quieren hacerlo sea problemática.

Apreciar la dimensión emancipatoria de esta dimensión del mercado, sin embargo, no debe llevarnos a negar su otra dimensión, su dimensión opresiva: el mercado es un contexto en 
el cual la necesidad del que necesita no cuenta, salvo en la medida en que ella cree una oportunidad a ser explotada por otro en su provecho. Dicho de otro modo, en el mercado cada uno recibe solamente aquello por lo que puedo pagar, y entonces la medida en que cada uno recibe es la medida en que puede pagar. Ese es el aspecto del mercado que es problemático, y sobre todo en materia de educación (y otros de los denominados "derechos sociales", como protección de la salud). Durante los últimos 30 años hemos entendido que es a través del mercado que lo que cada uno necesita debe ser obtenido, lo que quiere decir: aquello a lo cual cada uno tiene derecho es una provisión mínima (de educación, de salud, de seguridad social, etc), y sobre ese mínimo cada uno comprará lo que pueda en el mercado. En el caso de la educación, eso tiene el efecto perverso de que maximiza el aspecto deficitario del mercado (creando un sistema rigurosamente segregado) mientras minimiza su aspecto emancipador (porque dada la segregación del sistema educacional es falso que este provea de libertad de elegir).

Lo que a mi juicio dio contenido a las manifestaciones estudiantiles del año pasado fue que el mercado puede ser una manera adecuada de distribución de ciertos bienes, pero no para educación. Respecto, por ejemplo, de los automóviles el deber del Estado es sólo garantizar que ellos son de una calidad mínima (es decir, que satisfacen ciertos estándares de seguridad, contaminación etc), de modo que sobre ese mínimo cada uno comprará lo que pueda. Pero tratándose de educación (y la aplicabilidad de este argumento a la protección de la salud es demasiado obvia) la medida en que cada uno recibe no puede ser la medida en que cada uno puede pagar.

Habiendo distinguido de este modo las dos dimensiones del mercado, y habiendo identificado la dimensión que es problemática y las razones por las que es problemático que las universidades sean agentes de mercado, es ahora posible pensar un sistema que todavía descansara en el mercado en el primer sentido (que cada uno pueda elegir lo que quiere) pero que no descansara en el mercado en el segundo sentido (en que lo que cada uno recibe no esté vinculado a lo que pueda pagar). Esta idea se manifestó el año pasado en la demanda por gratuidad. Este no es, quizás, la mejor manera de identificar el contenido de la demanda, porque no se trata de que la educación sea "gratis" en el sentido de que nadie pague por ella. Se trata de neutralizar la segunda dimensión del mercado, rompiendo el vínculo que ella establece entre la provisión de educación y la medida en la que el que necesita educación puede pagar por ella.

Para romper este vínculo y así neutralizar la dimensión opresiva del mercado para maximizar su dimensión emancipadora es necesario un sistema de financiamiento conforme al cual la medida en que cada uno paga no sea la medida en que cada uno tiene, sino la medida en que cada uno puede pagar. La manera de hacerlo es a través del sistema tributario: cada uno paga lo que corresponde conforme a sus ingresos, y cada uno recibe en la medida en que necesita.

¿Es la competencia entre las universidades algo que debe, mediante regulación, ser eliminado? ¿Hay algunos aspectos de esa competencia que sean convenientes y otros que no los sean? ¿Cómo distinguirlios? 
Yo diría que parte de lo que significa introducir esta idea de un régimen de lo público implica pronunciarse en cuestiones como esta, sobre el sentido de la competencia entre universidades. Es claro que en muchas dimensiones la competencia entre instituciones es beneficiosa, pero hay otras en la que es claramente perjudicial. Una regulación razonable debe intentar distinguir estas dos dimensiones. A mi juicio, uno de los mejores ejemplos de la irracionalidad de la competencia es la enorme cantidad de recursos que se desperdician en publicidad. En una medida considerable, la publicidad tiene la peculiaridad de que es una situación de lo que en teoría de juegos se denomina "carrera armamentista". En una situación de ese tipo, una de las partes hace un gasto que la deja en mejor posición que la otra, lo que fuerza a la otra a hacer un gasto equivalente. A esto la primera responde haciendo un gasto adicional, que fuerza a la segunda a gastar de nuevo. Lo peculiar de esto es que el gasto de la segunda tiene por finalidad sólo neutralizar el gasto de la primera, por lo que al final de cada ciclo las dos partes se encuentran en la misma situación en la que se encontraban al principio, con la diferencia de que han hecho un gasto considerable. En una situación de este tipo sería preferible para ambas partes que ninguna de las dos gastara, pero esta es una decisión que no se puede tomar unilateralmente, porque el que decide no gastar por eso mismo pierde. La necesidad y conveniencia de la regulación aquí es evidente, porque si, por ejemplo, a ambas partes se les prohibiera gastar ambas quedarían en una mejor posición.

Este es sólo un ejemplo del modo en que la ausencia actual de regulación ha afectado el desarrollo de las instituciones universitarias, y que podría ser corregido mediante la regulación para evitar la irracionalidad que la competencia introduce sin afectar otras dimensiones en las que la competencia puede ser beneficiosa. La pregunta ha de ser siempre la misma: cómo configurar las condiciones en las que las universidades existen y se relacionan de modo de que el sistema universitario se desarrolle del modo que permita un desempeño más adecuado de su función. Es esta perspectiva del sistema la que ha sido sistemáticamente ignorada

Dentro del último tiempo se ha hablado mucho de esta noción de la universidad pública del siglo XXI, se ha discutido mucho en torno a esa noción, a si podemos hablar de universidad pública del Siglo XXI... ¿Tiene sentido la universidad pública en nuestra época?

Esa es una cuestión completamente crucial hoy, y por razones que son independiente de lo que hemos estado conversando. Es el cambio en la función que las universidades como instituciones desempeñan hoy que mencioné al principio. Su causa es la masificación de la educación universitaria.

Hoy estamos experimentando la transformación de una institución que se entendía a sí misma como la "conciencia crítica de la sociedad" o un espacio de búsqueda desinteresada de la verdad a una que se entiende primariamente como una fábrica de profesionales. ¿Es esta transformación algo que debe ser celebrado y fomentado? Creo que hay que responder esto con 
cautela. No tengo duda alguna de que, en principio, el hecho de que la educación universitaria esté al alcance de personas que en el pasado no habrían podido acceder a ella es algo que debe ser celebrado. Hoy tenemos, por las razones que hemos estado discutiendo, problemas graves con la calidad de la educación que muchas de ellas ofrecen y también por las deudas asumidas por los estudiantes, pero esos son problemas que deben ser enfrentados precisamente porque la otra cara de esa situación: el hecho de que muchos más pueden acceder hoy a la universidad que antes, es importante y valioso.

Pero este mismo fenómeno, que debe ser celebrado sin reservas, lleva a una cuestión distinta, la transformación de la institución universitaria a la que aludía más arriba. Y esta transformación plante la siguiente pregunta: ¿pueden las instituciones que se entienden como fábricas de profesionales desempeñar las funciones que la universidad desempeñaba en el pasado? Y si la respuesta a esta pregunta es negativa, ¿qué ocurre con esas funciones? ¿Quién las desempeña?

Yo no tengo una respuesta para estas preguntas, pero si creo que el hecho de que entre las universidad de mayor prestigio haya universidades estatales es algo que será extraordinariamente importante cuando ellas surjan y deban ser respondidas. Esto porque un sistema universitario se construye, en parte, sobre la base de instituciones que resultan paradigmáticas, que reflejan de modo más completo la idea de una la institución. En el pasado, algunos liceos públicos (los que hoy son "emblemáticos") desempeñaban esa función paradigmática respecto de la educación escolar, y ellos entonces orientaban el desarrollo de todos los establecimientos. En el sistema escolar eso cambió, y hoy los establecimientos más destacados ya no son establecimientos públicos, lo que disminuye la posibilidad de guiar el desarrollo del sistema hacia una autocomprensión substantivamente pública. El mismo proceso, a mi juicio, está hoy en curso en el sistema universitario, aunque por las características de éste está en una etapa todavía temprana. Eso da una oportunidad fundamental para poder por lo menos modular algunas de las consecuencias de la transformación de la universidad en fábricas de profesionales, porque permite que a través del modelo provisto por una universidad pública como la Universidad de Chile ese proceso sea tematizado teniendo en vista el interés público, y no sólo intereses privados.

En este contexto, la pregunta por el sentido de las universidades estatales se hace más urgente, y parte de la respuesta a esa pregunta es la oportunidad invaluable que representa el hecho de que las universidades estatales todavía no se hayan transformado en universidades "emblemáticas" pero solo emblemáticas como los liceos "emblemáticos", sino que son todavía instituciones que sirven de ejemplos para el desarrollo de las demás instituciones. Pero como ese proceso está hoy en curso, es imperativo actuar ahora para abortarlo. 